\title{
Endothelium-derived Relaxing Factor/Nitric Oxide Modulates Angiotensin II Action in the Isolated Microperfused Rabbit Afferent but Not Efferent Arteriole
}

\author{
Sadayoshi Ito, Shuji Arima, Yi Lin Ren, Luis A. Juncos, and Oscar A. Carretero \\ Hypertension and Vascular Research Division, Department of Medicine and \\ Heart and Vascular Institute, Henry Ford Hospital, Detroit, Michigan 48202
}

\begin{abstract}
It has been reported that sensitivity to angiotensin II (Ang II) is higher in efferent (Ef) than afferent (Af) arterioles (Arts). We tested the hypothesis that this is due to arteriolar differences in the interaction between Ang II and endothelium-derived relaxing factor/nitric oxide (EDNO). Rabbit Af-Arts with glomerulus intact were microperfused in vitro at a constant pressure. Ef-Arts were perfused from the distal end of either the Af-Art (orthograde perfusion) or the Ef-Art (retrograde perfusion) to eliminate influences of the Af-Art or glomerulus, respectively. Ang II did not alter Af-Art luminal diameter until the concentration reached $10^{-9} \mathrm{M}$, which decreased the diameter by $11 \pm 2.6 \%(n=11 ; P<0.002)$. In contrast, Ef-Arts became significantly constricted at concentrations as low as $10^{-11}$ $M$ with either perfusion. Surprisingly, the decrease in Ef-Art diameter at $10^{-10}, 10^{-9}$, and $10^{-8} \mathrm{M}$ was significantly greater with retrograde perfusion $(44 \pm 6.9 \%, 70 \pm 5.6 \%$, and $74 \pm 4.1 \%$, respectively; $n=5$ ) than with orthograde perfusion ( $16 \pm 4.2 \%$, $25 \pm 2.9 \%$, and $35 \pm 3.5 \% ; n=9)$. ENDO synthesis inhibition with $10^{-4} \mathrm{M}$ nitro-L-arginine methyl ester (L-NAME) decreased the diameter to a greater extent in Af-Arts (22 $\pm 3.0 \%$; $n$ $=11)$ compared to Ef-Arts with either orthograde (9.5 $\pm 2.3 \%$; $n=8)$ or retrograde perfusion $(1.2 \pm 2.1 \% ; n=6)$. With LNAME pretreatment, Af-Art constriction induced by $10^{-10} \mathrm{M}$ $(14 \pm 4.0 \%, n=9)$ and $10^{-9} M$ Ang II $(38 \pm 3.9 \%)$ was significantly greater compared to nontreated Af-Arts. In contrast, $L$ NAME pretreatment had no effect on Ang II-induced constriction in Ef-Arts with either perfusion. In conclusion, this study demonstrates higher sensitivity of Ef-Arts to Ang II, particularly with retrograde perfusion. Our results suggest that EDNO significantly modulates the vasoconstrictor action of Ang II in Af-Arts II but not Ef-Arts, contributing to the differential sensitivity to Ang II. (J. Clin. Invest. 1993. 91:20122019.) Key words: glomerular hemodynamics $\bullet$ nitro-L-arginine methyl ester • renal microcirculation • sensitivity
\end{abstract}

\section{Introduction}

The renin-angiotensin system plays an important role in the regulation of blood pressure, homeostasis of fluid volume and electrolytes, and pathogenesis of various forms of hyperten-

Address reprint requests to Dr. Sadayoshi Ito, Hypertension and Vascular Research Division, Henry Ford Hospital, 2799 West Grand Boulevard, Detroit, MI 48202.

Received for publication 8 September 1992 and in revised form 18 December 1992.

J. Clin. Invest.

(c) The American Society for Clinical Investigation, Inc.

0021-9738/93/05/2012/08 \$2.00

Volume 91, May 1993, 2012-2019 sion. A number of studies have examined the role of angiotensin II (Ang II), ${ }^{1}$ the physiologically active component of the system, in the control of renal hemodynamics as well as its sites of action within the kidney (1). Infusion of Ang II or inhibition of its action in the kidney has been shown to result in an increased or decreased filtration fraction, respectively (2-5). Thus it has been postulated that sensitivity to Ang II is higher in the postglomerular efferent (Ef) than in the preglomerular afferent (Af) arteriole (Art). Although recent studies using isolated microvessels have demonstrated higher sensitivity of the Ef-Art $(6,7)$, the mechanism(s) responsible for this difference in sensitivity is (are) not well understood.

We have recently shown that in isolated microperfused rabbit Af-Arts, inhibition of endothelium-derived relaxing factor/ nitric oxide (EDNO) not only reduces basal luminal diameter but also augments the vasoconstrictor action of Ang II (8). This suggests that EDNO, which is produced locally in the Af-Art, is an important autacoid hormone that controls vascular reactivity. Although studies have shown that endogenous EDNO plays an important role in the control of renal hemodynamics (9), it is not known whether either activity of EDNO or its interaction with other vasoactive substances differs between the Af-Art and Ef-Art. Since the Af-Art and Ef-Art are crucial vascular segments that control glomerular hemodynamics (and hence renal excretory function), it would be important to understand the action of EDNO in these vessels.

In the present study, we tested the hypothesis that endogenous EDNO modulates Ang II action to a greater extent in the Af-Art than in the Ef-Art, contributing to the difference in sensitivity to Ang II. For this, we microdissected and perfused rabbit Af-Arts and Ef-Arts in vitro and studied whether $(a)$ response to EDNO inhibition or (b) EDNO modulation of Ang II action is different between the two vessels.

\section{Methods}

Isolation and microperfusion of the rabbit Af- and Ef-Art

We used a method similar to that described previously to isolate and microperfuse Af-Arts $(8,10)$. Briefly, young male New Zealand white rabbits (1.5-2.0 kg), fed standard rabbit Chow (Ralston Purina Co., St. Louis, MO) and tap water ad lib., were anesthetized with intravenous sodium pentobarbital $(40 \mathrm{mg} / \mathrm{kg})$ and given an intravenous injection of heparin $(500 \mathrm{U})$. The kidneys were removed and sliced along the corticomedullary axis. Slices were placed in ice-cold MEM (Gibco Laboratories, Grand Island, NY) containing 5\% BSA (Sigma Chemical Co., St. Louis, MO) and dissected under a stereomicroscope (model SZH; Olympus Corp., Tokyo, Japan) as described previously. From

1. Abbreviations used in this paper: Af-Art and Ef-Art, afferent and efferent arteriole, respectively; Ang II, angiotensin II; EDNO, endothelium-derived relaxing factor/nitric oxide; L-NAME, nitro-L-arginine methyl ester; NE, norepinephrine. 
each rabbit, a single superficial Af-Art with its glomerulus intact was microdissected. Using a micropipette, the Af-Art was transferred to a temperature-regulated chamber mounted on an inverted microscope (model IMT-2; Olympus Corp.) with Hoffman modulation. The AfArt was then cannulated with an array of glass pipettes as described previously $(8,10)$. Intraluminal pressure was measured by Landis' technique, using a fine pipette introduced into the arteriole through the perfusion pipette. This technique was used because it has been reported that pressure measurement by a servo-null system is not accurate with our arrangement of pipettes. The arteriole was perfused with oxygenated medium 199 containing 5\% BSA, and intraluminal pressure was maintained at $60 \mathrm{mmHg}$ throughout the experiment.

We employed two approaches to study Ef-Art, namely orthograde and retrograde perfusion. For orthograde perfusion, a short $(\sim 50 \mu \mathrm{m})$ Af-Art with its glomerulus intact and a segment of the attached Ef-Art (250-300 $\mu \mathrm{m}$ ) were microdissected. The Af-Art was cannulated as described above, except that the perfusion pipette was advanced to the end of the Af-Art (Fig. 1, top). The tip of the pressure pipette was placed just beyond the distal end of the Af-Art, and intraluminal pressure at this point was maintained at $50 \mathrm{mmHg}$ throughout the experiment so as to eliminate the hemodynamic influences of the Af-Art. In five experiments, we measured pressure in the Ef-Art. The Ef-Art was drawn into a holding pipette (i.d., $20 \mu \mathrm{m}$ ) which had a slightly negative internal pressure $(-2 \mathrm{mmHg})$. A pressure pipette (o.d., $2 \mu \mathrm{m})$ was inserted into the Ef-Art from the distal end and advanced until its tip reached a point $\sim 50 \mu \mathrm{m}$ distal to the glomerulus. In that only a $2-\mu \mathrm{m}$ pressure pipette was inserted into the Ef-Art with a luminal diameter of $\sim 15 \mu \mathrm{m}$ (thus the pipette occupied only $2 \%$ of the cross-sectional

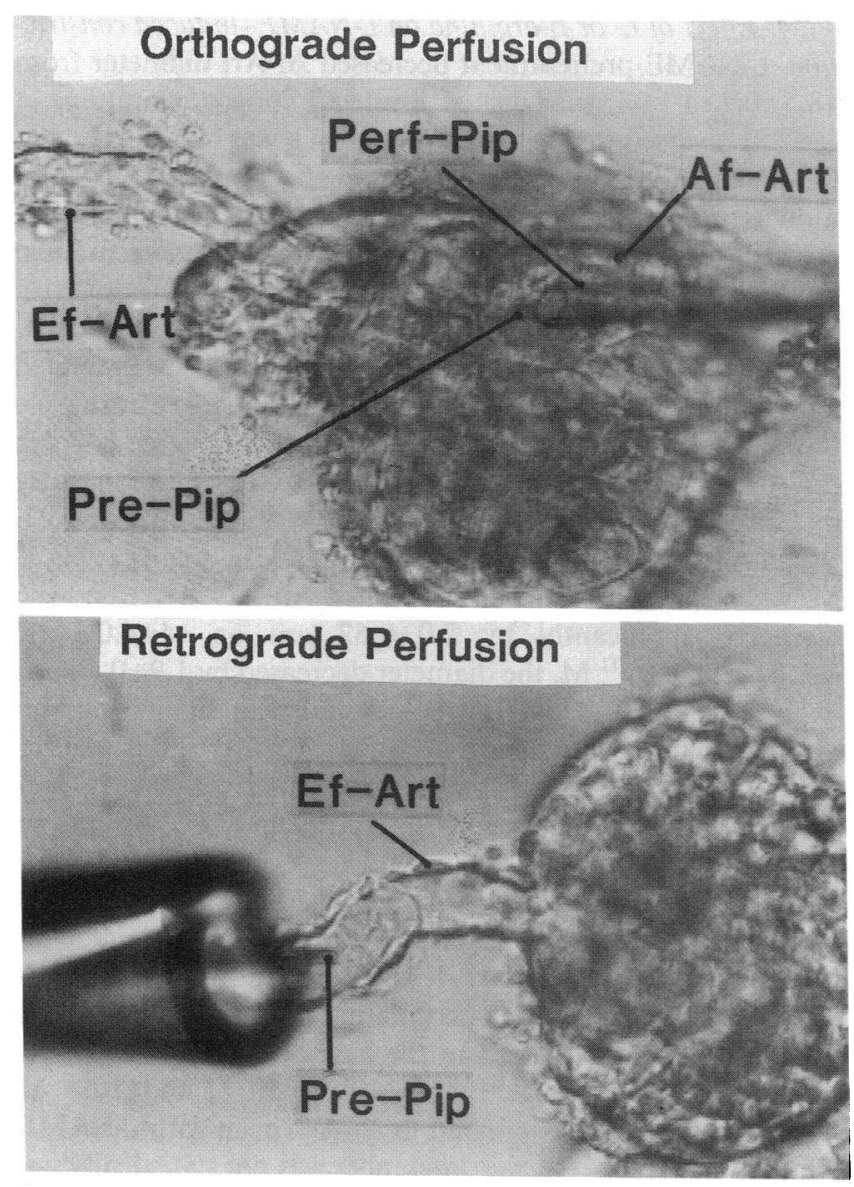

Figure 1. Efferent arteriole ( $E f-A r t)$ perfused in orthograde (top) and retrograde direction (bottom). Perf-Pip, perfusion pipette; Pre-Pip, pressure pipette; Af-Art, afferent arteriole. area), perfusate flowed freely into the holding pipette. The pressure measured at this point varied between 32 and $41 \mathrm{mmHg}$, with an average of $36.4 \pm 1.3 \mathrm{mmHg}$. For retrograde perfusion, most of the Af-Art was removed and the Ef-Art ( $100-150 \mu \mathrm{m})$ perfused from its distal end at $35 \mathrm{mmHg}$ throughout the experiment (Fig. 1, bottom). In this preparation, there are no influences of either hemodynamic changes induced by the glomerulus or vasoactive substances produced by the glomerulus/Af-Art.

The bath, which was identical to the arteriolar perfusate except that it contained $0.1 \%$ BSA, was exchanged continuously. Microdissection and cannulation of the arteriole were completed within $90 \mathrm{~min}$ at $8^{\circ} \mathrm{C}$, after which the bath was gradually warmed to $37^{\circ} \mathrm{C}$ for the rest of the experiment. Once the temperature was stable, a 30 -min equilibration period was allowed before taking any measurements. Images of the arteriole were displayed at magnifications up to $\times 1,980$ and recorded with a video system consisting of a camera (model DXC-755; Sony, Tokyo, Japan), monitor (model PVM1942Q; Sony) and video recorder (model EDV-9500; Sony). The diameter at the most constricted point (which was seen at a segment within $50 \mu \mathrm{m}$ from the glomerulus) was measured with an image analysis system (Fryer, Carpentersville, IL).

\section{Experimental protocols}

1. Response to Ang II. After the 30-min equilibration period, increasing doses of Ang II ( $10^{-11}$ to $10^{-8} \mathrm{M}$; Sigma Chemical Co.) were added to the bath. Luminal diameter was measured immediately before adding Ang II and observed for at least $3 \mathrm{~min}$ at each dose.

2. Response to norepinephrine ( $N E$ ). To examine whether differences in sensitivity between the Af- and Ef-Art are common to all vasoconstrictors, we compared the effect of $\mathrm{NE}$ on luminal diameter of Af- and Ef-Arts. After the equilibration period, increasing doses of NE $\left(10^{-8}\right.$ to $10^{-6} \mathrm{M}$; Sigma Chemical Co.) were added to the bath. Luminal diameter was measured immediately before adding NE and observed for at least $3 \mathrm{~min}$ at each dose.

3. Inhibition of EDNO synthesis with nitro-L-arginine methyl ester ( $L-N A M E)$. After the equilibration period, increasing doses of $\mathrm{L}$ NAME $\left(10^{-7}\right.$ to $10^{-4} \mathrm{M}$; Sigma Chemical Co.), a compound that inhibits EDNO synthesis (11), were added to the lumen. Luminal diameter was measured immediately before adding L-NAME and observed for at least $15 \mathrm{~min}$ at each dose.

4. Effect of L- or D-arginine on L-NAME-induced constriction. We examined whether Af-Art vasoconstriction induced by L-NAME is specifically due to inhibition of EDNO synthesis from L-arginine (12). We first inhibited EDNO synthesis with L-NAME at $10^{-4} \mathrm{M}$, after which either L-arginine or its inactive isomer D-arginine at $10^{-3} \mathrm{M}$ (Sigma Chemical Co.) was added to the lumen together with L-NAME.

5. Effect of L-NAME on Ang II-induced vasoconstriction. After the equilibration period, $10^{-4} \mathrm{M}$ L-NAME was added to the arteriolar perfusate. Fifteen minutes later, the effect of Ang II was examined as in protocol 1 . We have previously shown that L-NAME at this concentration blocks acetylcholine-induced vasodilation in Af-Arts preconstricted with NE (8). We have also confirmed that L-NAME is just as effective in the Ef-Art. The Ef-Arts were preconstricted with NE ( 5 $\times 10^{-7} \mathrm{M}$ ) to $82 \pm 3.6 \%$ of basal diameter in orthograde perfusion ( $n$ $=5$ ) and $46 \pm 6.5 \%$ in retrograde perfusion $(n=5)$, while intraluminal addition of acetylcholine $\left(10^{-5} \mathrm{M}\right)$ reversed the diameter to $105 \pm 7.1 \%$ and $106 \pm 5.6 \%$ of basal values, respectively. When NE was given to Ef-Arts pretreated with L-NAME, the diameter decreased to $79 \pm 4.7 \%$ of baseline in orthograde perfusion $(n=6)$ and $63 \pm 12 \%$ in retrograde perfusion $(n=4)$, which remained unchanged after addition of acetylcholine ( $80 \pm 4.6 \%$ and $64 \pm 12 \%$, respectively). (See Results and Discussion for weaker responses to $\mathrm{NE}$ in orthograde than in retrograde perfusion.)

6. Effect of L-NAME on NE-induced vasoconstriction. We examined whether L-NAME-induced enhancement of vasoconstrictor action is common to all vasoconstrictors. The experimental design was the same as in protocol 5, except that $\mathrm{NE}\left(10^{-8}\right.$ to $\left.10^{-6} \mathrm{M}\right)$ was used instead of Ang II. 
7. Effect of NE pretreatment on Ang II-induced vasoconstriction. Since L-NAME not only augmented Ang II-induced vasoconstriction in Af-Arts but also reduced basal diameter (see Results), we tested whether L-NAME affected the action of Ang II by increasing basal tone. The experimental design was the same as in protocol 5, except that we used only Af-Arts and reduced basal diameter with $10^{-7} \mathrm{M}$ NE instead of $10^{-4} \mathrm{M}$ L-NAME.

\section{Statistics}

Values were expressed as mean \pm SEM, and all statistical analyses were done using absolute values. A paired $t$ test was used to examine whether the diameter at a given concentration was different from the control value. Analysis of covariance (ANCOVA) was used to examine whether the change in diameter at a given concentration was different between groups. For both analyses, $P<0.0125(0.05 / 4$; Bonferroni adjustment) was considered significant.

\section{Results}

1. Response to Ang II. Basal luminal diameter of the Af-Arts was $16.1 \pm 0.9 \mu \mathrm{m}(n=11)$, while that of the Ef-Arts was $15.7 \pm 0.6 \mu \mathrm{m}$ with orthograde perfusion $(n=9)$ and $16.3 \pm 1.3$ $\mu \mathrm{m}$ with retrograde perfusion $(n=5)$. As shown in Fig. 2 , Ang II had no effect on Af-Arts until the concentration reached $10^{-9} \mathrm{M}$, which decreased luminal diameter by $2.0 \pm 0.45 \mu \mathrm{m}$ or $11 \pm 2.6 \%(P<0.002)$; at $10^{-8} \mathrm{M}$, the decrease was $6.7 \pm 1.1 \mu \mathrm{m}$ or $40 \pm 5.3 \%(P<0.0001)$. In contrast, in Ef-Arts perfused in either direction, Ang II began to cause significant constriction at concentrations as low as $10^{-11} \mathrm{M}$. In orthograde perfusion, the decrease in diameter induced by Ang II was $1.8 \pm 0.55 \mu \mathrm{m}$ or $11 \pm 3.8 \%$ at $10^{-11} \mathrm{M}(P<0.01), 2.4 \pm 0.61 \mu \mathrm{m}$ or $16 \pm 4.2 \%$ at $10^{-10} \mathrm{M}(P<0.005), 3.9 \pm 0.39 \mu \mathrm{m}$ or $25 \pm 2.9 \%$ at $10^{-9} \mathrm{M}(P$ $<0.0001)$, and $5.5 \pm 0.57 \mu \mathrm{m}$ or $35 \pm 3.5 \%$ at $10^{-8} \mathrm{M}(P$ $<0.0001)$; in retrograde perfusion, the corresponding values were $1.6 \pm 0.25 \mu \mathrm{m}$ or $10 \pm 2.1 \%(P<0.005), 7.1 \pm 1.11 \mu \mathrm{m}$ or $44 \pm 6.9 \%(P<0.003), 11.2 \pm 0.68 \mu \mathrm{m}$ or $70 \pm 5.7 \% \quad(P$ $<0.0001)$, and $11.8 \pm 0.76 \mu \mathrm{m}$ or $74 \pm 4.1 \%(P<0.0001)$. Compared to the Af-Arts, the decrease in Ef-Art diameter was significantly greater at doses of $10^{-10}$ and $10^{-9}$ in orthograde perfusion and at all doses examined in retrograde perfusion. In addition, the decrease in Ef-Art diameter induced by $10^{-10}$ to

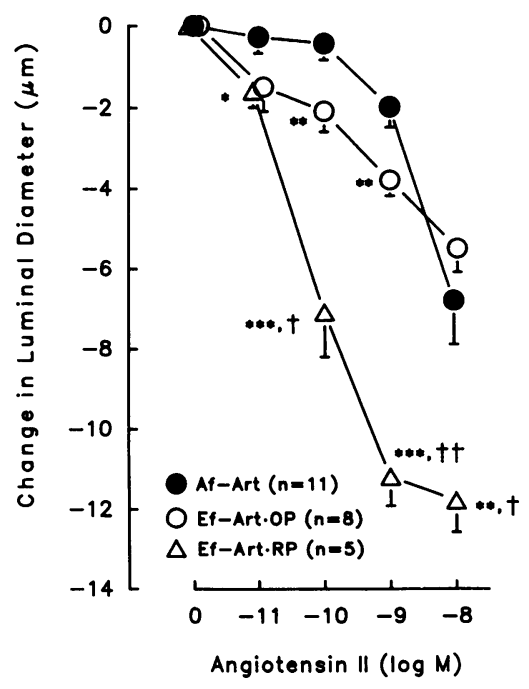

Figure 2. Change in luminal diameter induced by Ang II in microperfused afferent arterioles $(A f-A r t)$ and efferent arterioles studied with orthograde $(E f$ $A r t \cdot O P)$ or retrograde perfusion $(E f-A r t \cdot R P)$. $*, * * * * * P<0.012$ 0.003 , and 0.0001 , respectively, compared with Af-Art. ${ }^{+,++} P$ $<0.003$ and 0.0001 , respectively, compared with Ef-Art·OP.
$10^{-8} \mathrm{M}$ Ang II was significantly greater in retrograde than in orthograde perfusion (Fig. 2).

2. Response to NE. Basal luminal diameter of the Af-Arts was $16.2 \pm 0.9 \mu \mathrm{m}(n=7)$, while that of the Ef-Arts was $14.8 \pm 0.5 \mu \mathrm{m}$ with orthograde perfusion $(n=9)$ and $14.5 \pm 1.4$ $\mu \mathrm{m}$ with retrograde perfusion $(n=5)$. In both the Af-Arts and the Ef-Arts with retrograde perfusion, NE decreased luminal diameter in a similar dose-dependent manner, with significant constriction occurring at $10^{-7} \mathrm{M}$ (Fig. 3). In the Ef-Arts studied with orthograde perfusion, $\mathrm{NE}$ at $10^{-8}$ and $10^{-7} \mathrm{M}$ induced similar responses compared to either Af-Arts or Ef-Arts with retrograde perfusion; however, NE at higher concentrations induced significantly weaker constriction.

3. Inhibition of EDNO synthesis with L-NAME. Basal luminal diameter of the Af-Arts was $17.4 \pm 0.8 \mu \mathrm{m}(n=11)$, while that of Ef-Arts was $14.7 \pm 0.7 \mu \mathrm{m}$ with orthograde perfusion ( $n$ $=8$ ) and $15.2 \pm 0.72 \mu \mathrm{m}$ with retrograde perfusion $(n=6)$. As shown in Fig. 4, L-NAME decreased Af-Art diameter in a dosedependent manner, with the decrease becoming $2.6 \pm 0.76 \mu \mathrm{m}$ or $14 \pm 4.3 \%$ at $10^{-5} \mathrm{M}(P<0.01)$ and $3.9 \pm 0.58 \mu \mathrm{m}$ or $22 \pm 3.0 \%$ at $10^{-4} \mathrm{M}(P<0.0001)$. With orthograde perfusion, $10^{-4} \mathrm{M}$ L-NAME caused a statistically significant decrease in Ef-Art diameter $(1.4 \pm 0.33 \mu \mathrm{m}$ or $9.5 \pm 2.3 \% ; P<0.01)$; however, it tended to be smaller than in the Af-Arts $(P=0.05)$. Moreover, with retrograde perfusion, L-NAME had no effect on Ef-Arts at any dose, and the decrease in diameter at $10^{-4} \mathrm{M}$ was significantly different compared to the Af-Arts ( $P$ $<0.005$ )

4. Effect of $L$ - or D-arginine on $L-N A M E$-induced constriction. L-NAME pretreatment decreased Af-Art diameter from $18.5 \pm 0.7$ to $14.8 \pm 0.7(n=6)$, while addition of L-arginine at $10^{-3} \mathrm{M}$ reversed the diameter to control levels $(19.4 \pm 1.1 \mu \mathrm{m})$. On the other hand, D-arginine had no effect on the L-NAMEinduced decrease in diameter; with L-NAME, the diameter decreased from $18.7 \pm 0.7$ to $15.0 \pm 0.8 \mu \mathrm{m}(n=4)$ and remained unchanged after addition of D-arginine $(14.4 \pm 1.1 \mu \mathrm{m})$.

5. Effect of L-NAME on Ang II-induced vasoconstriction. Changes in diameter induced by Ang II in L-NAME-treated and nontreated Af-Arts are depicted in Fig. 5, while an example of the responses observed at $10^{-9} \mathrm{M}$ is shown in Fig. 6. After L-NAME pretreatment, basal diameter decreased significantly by $22 \pm 5.6 \%$, falling from $17.3 \pm 1.0$ to $13.2 \pm 0.8 \mu \mathrm{m}(n=9)$. When Af-Arts were pretreated with L-NAME, Ang II at $10^{-10}$ $M$, which had no effect on nontreated Af-Arts, decreased the diameter significantly by $2.0 \pm 0.62 \mu \mathrm{m}$ or $14 \pm 4.0 \%(P$ $<0.012$ ); at $10^{-11} \mathrm{M}$, the diameter decreased by $1.9 \pm 0.66 \mu \mathrm{m}$ or $13 \pm 4.2 \%$, but it did not reach statistical significance $(P$ $=0.02$ ). When compared to non-treated Af-Arts, the Ang IIinduced decrease in diameter was greater in the L-NAME-pretreated Af-Arts at doses of $10^{-11}, 10^{-10}$, and $10^{-9}$ but not at $10^{-8} \mathrm{M}$.

In contrast, $\mathrm{L}-\mathrm{NAME}$ pretreatment had no effect on Ef-Arts whether they were perfused in an orthograde or retrograde direction (Fig. 7). Thus, with L-NAME pretreatment, Ang II-induced constriction became no different in Af-Arts and Ef-Arts with orthograde perfusion. In the case of Ef-Arts with retrograde perfusion, constriction at $10^{-10}, 10^{-9}$, and $10^{-8} \mathrm{M}$ seemed to be greater compared to Af-Arts even with L-NAME pretreatment; however, the differences was not statistically significant $(P>0.4)$.

6. Effect of L-NAME on NE-induced vasoconstriction. LNAME pretreatment decreased luminal diameter by $25 \pm 3.4 \%$, 


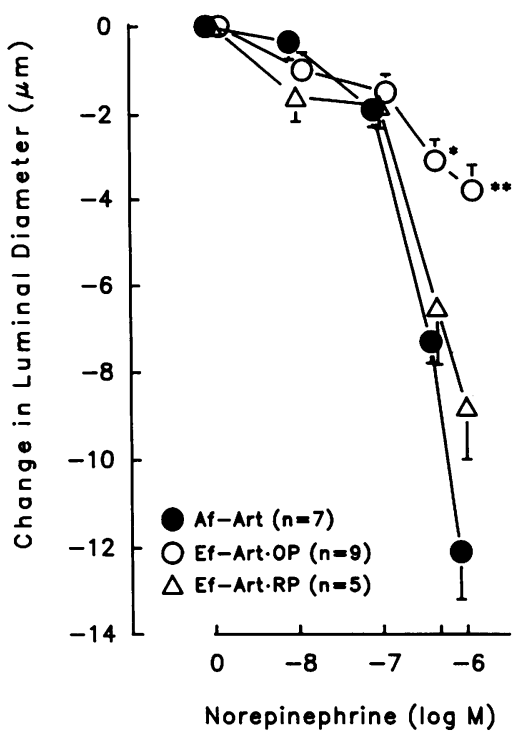

from $20.5 \pm 1.0$ to $15.7 \pm 1.1 \mu \mathrm{m}(n=6)$. However, it did not affect NE-induced constriction in Af-Art (Fig. 8), in marked contrast to the augmented action of Ang II (Figs. 5 and 6 ).

7. Effect of NE pretreatment on Ang II-induced vasoconstriction. Pretreatment with NE reduced basal diameter by $16 \pm 2.8 \%$, from $17.3 \pm 0.8$ to $14.6 \pm 0.9 \mu \mathrm{m}(n=10)$, which was no different from that induced by L-NAME. However, it did not affect the vasoconstrictor action of Ang II (Fig. 9).

\section{Discussion}

Differential sensitivity of the pre- and postglomerular arterial vessels to Ang II has been of great interest to many investigators. Using various experimental models, researchers have reported that sensitivity to Ang II is higher $(3,7,13,14)$ or exclusively present in the postglomerular vessels $(2,6,15,16)$, or similar in both vessels (17-20). To avoid neurohormonal and systemic hemodynamic influences, Edwards (6) developed an in vitro preparation consisting of an isolated rabbit

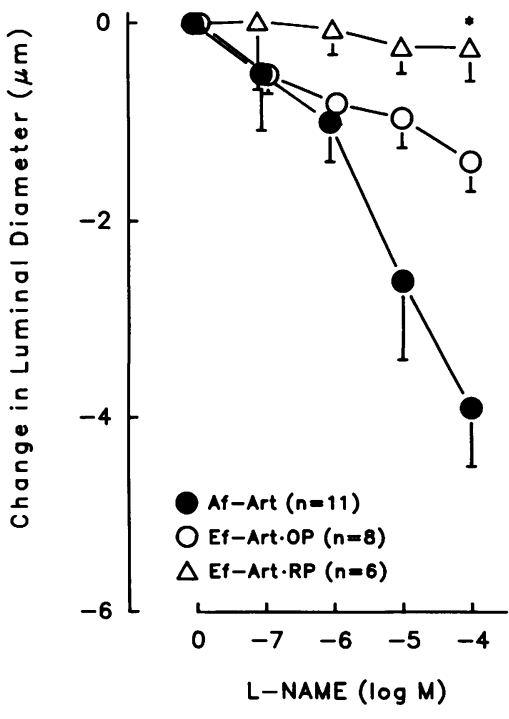

Figure 4. Change in luminal diameter induced by L-NAME, which inhibits synthesis of EDNO, in microperfused afferent arterioles $(A f-A r t)$ and efferent arterioles studied with orthograde ( $E f$ $A r t \cdot O P)$ or retrograde perfusion $(E f-A r t \cdot R P)$ ${ }^{*} P<0.005$ compared with Af-Art.

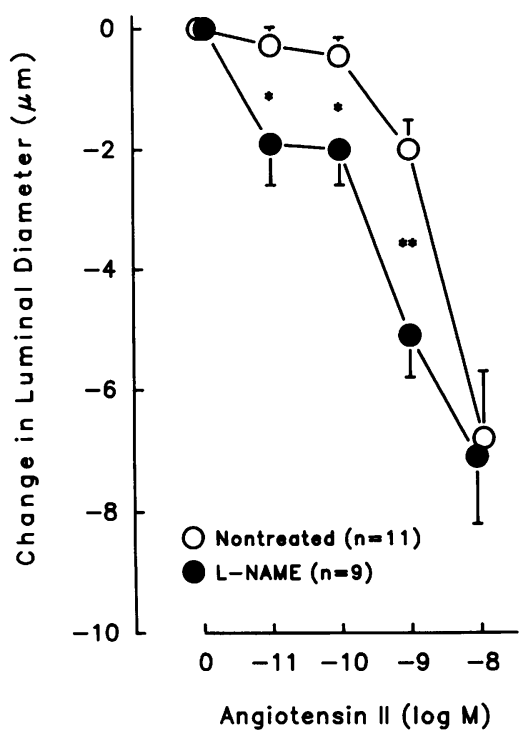

Figure 5. Change in luminal diameter induced by Ang II in nontreated afferent arterioles (Nontreated ) and afferent arterioles pretreated with L-NAME. ${ }^{*, * *} P$ $<0.002$ and 0.0001 , respectively, for nontreated versus L-NAME.

renal microvascular segment with one end cannulated and the other occluded. He reported that, although Ang II caused dosedependent vasoconstriction in the Ef-Art, the Af-Art was completely nonresponsive to Ang II even at $10^{-6} \mathrm{M}$. Using a similar technique in rats, Yuan et al. (7) reported that Ang II causes vasoconstriction in both Af-Art and Ef-Art, but with a lower $\mathrm{EC}_{50}$ in Ef-Art. Our results are qualitatively consistent with these in vitro studies, even though there was a clear difference in the vascular responses of the Ef-Arts with orthograde versus retrograde perfusion. Nevertheless, our observations, taken together with previous studies, clearly demonstrate that sensitivity to Ang II is higher in Ef-Arts than in Af-Arts.

We found striking differences in the vascular reactivity of Ef-Arts with orthograde versus retrograde perfusion. In retrograde perfusion, both Ang II- and NE-induced vasoconstriction were very strong, and the dose-response curves obtained were almost identical to those reported by Edwards (6) and Yuan et al. (7) in isolated but nonperfused Ef-Arts. On the other hand, vasoconstriction was much weaker in orthograde perfusion. Thus, when Ang II action on Af-Arts and Ef-Arts was compared, the difference was less marked (albeit significant) with orthograde than with retrograde perfusion of the Ef-Arts. The reason for the differences in Ef-Art reactivity is not clear. It has been reported that Ef-Art response to Ang II but not NE is pressure dependent (21). However, we observed that not only the response to Ang II but also that to NE (which is pressure independent) were clearly different with orthograde versus retrograde perfusion. Furthermore, when we measured pressure in Ef-Arts studied with orthograde perfusion, it was found to be similar to that used for retrograde perfusion. The fact that L-NAME did not affect the differences in Ef-Art reactivity suggests that factors other than EDNO may be involved. It may be that in orthograde perfusion, the release of other vasoactive substances ( such as prostaglandins) by the glomerulus (22) could influence the Ef-Art response. The differences may also be related to shear stress. Due to ultrafiltration at the glomerulus, protein concentration in the Ef-Art (and hence shear stress) would be higher in orthograde than retrograde perfusion. This difference as well as the direction and rate of flow may have affected synthesis of various vasoactive substances by the endothelium $(23,24)$. 

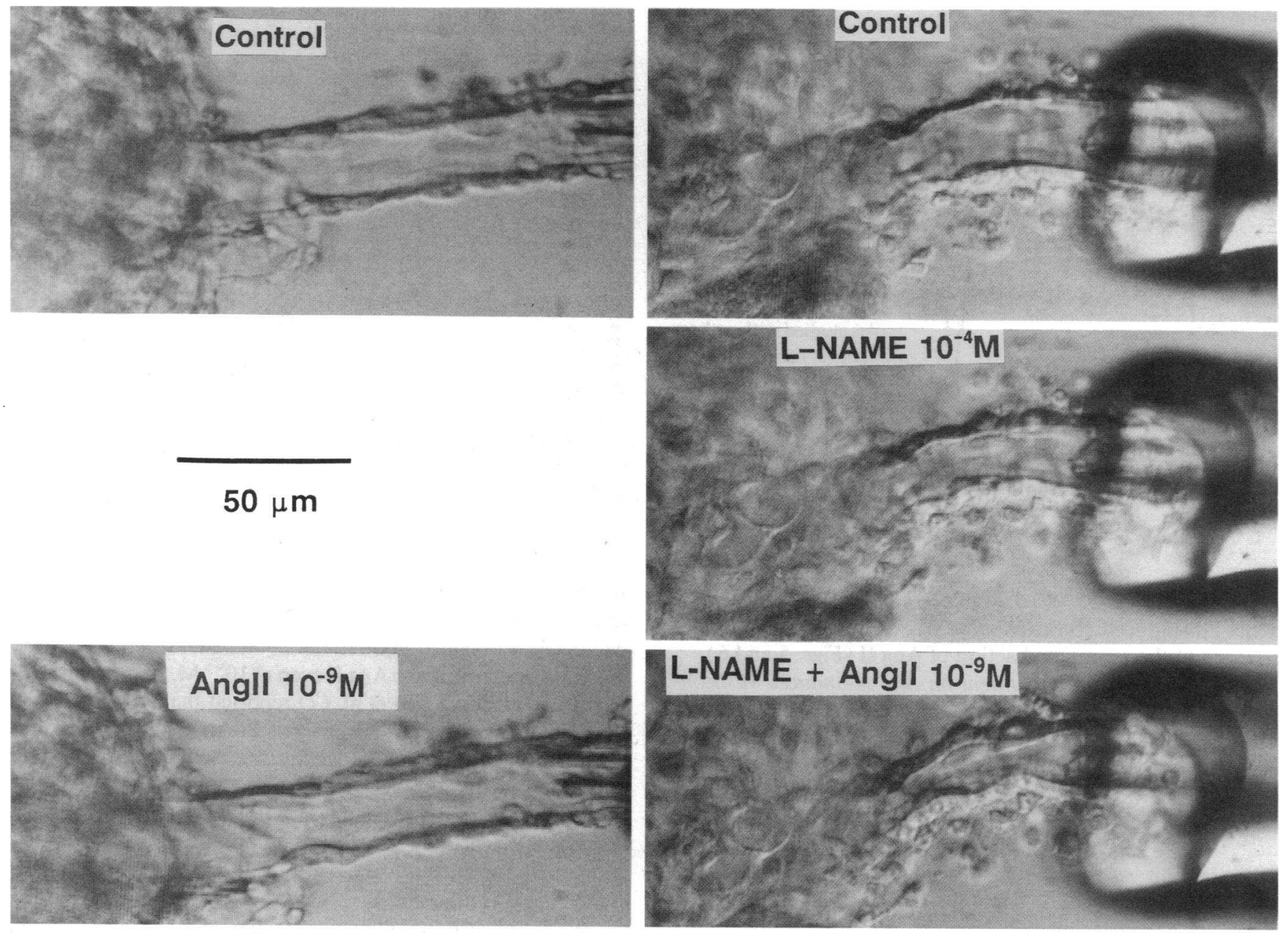

Figure 6. Vasoconstriction induced by $10^{-9} \mathrm{M}$ angiotensin II (AngII) in a nontreated afferent arteriole (left) and afferent arteriole pretreated with L-NAME (right). Note that L-NAME not only reduced basal diameter but augmented the vasoconstrictor action of angiotensin II.

In contrast to the difference in sensitivity to Ang II, NE has been reported to have a similar effect on isolated Af-Arts and Ef-Arts $(6,7)$. Consistent with these studies, we found no difference in NE-induced vasoconstriction between Af-Arts and Ef-Arts with retrograde perfusion. However, in the Ef-Arts studied with orthograde perfusion, the decreases in diameter induced by higher concentrations $\left(5 \times 10^{-7}\right.$ and $\left.10^{-6} \mathrm{M}\right)$ were significantly less compared to either Af-Arts or Ef-Arts with retrograde perfusion. Weaker responses of Ef-Arts than Af-Arts to NE or electrical stimulation of renal nerves have also been observed in rat juxtamedullary nephrons (P. K. Carmines, personal communication ) and the isolated perfused hydronephrotic kidney (25). The fact that NE-induced constriction of EfArts is weaker or at best similar compared to Af-Arts suggests that the Ef-Art's higher sensitivity to Ang II is not due to either structural or physical factors, such as its thinner arteriolar wall or lower perfusion pressure. In fact, Yuan et al. (7) have shown that reducing intraluminal pressure of the Af-Art from 90 to 30 $\mathrm{mmHg}$ did not increase sensitivity to Ang II. They also reported that indomethacin had no effect on the Af-Art, suggesting that prostaglandins are not involved in the difference in sensitivity.

We have recently presented evidence that endogenous production of EDNO is important in the control of Af-Art reactiv- ity (8). We have shown that Ang II at $10^{-7} \mathrm{M}$ causes only transient constriction of microperfused rabbit Af-Arts, which becomes stronger and more persistent when they are pretreated with L-NAME. These effects of L-NAME are most likely due to inhibition of EDNO, since the present study has shown that L-arginine (the precursor of EDNO) but not D-arginine (an inactive isomer) reverses L-NAME-induced constriction of the Af-Art. We have now extended our previous findings, showing that L-NAME pretreatment increases sensitivity of the Af-Art to Ang II at more physiological concentrations. It is unlikely that this enhancement is due to the change in basal tone, since a similar reduction in basal diameter with NE had no effect on Ang II-induced vasoconstriction. In addition, L-NAME pretreatment did not alter Af-Art responses to NE, showing that enhancement of vasoconstriction by L-NAME is not common to all vasoconstrictors. On the other hand, L-NAME pretreatment had no effect on Ef-Art response to Ang II whether it was studied with orthograde or retrograde perfusion. Furthermore, we found that the L-NAME-induced decrease in basal luminal diameter is much smaller in Ef-Arts than in Af-Arts. These results suggest that in the Af-Art, endogenous EDNO not only controls basal tone but also counteracts the vasoconstrictor action of Ang II (but not NE), whereas it plays a minimal role in the Ef-Art. Thus the difference in sensitivity to Ang II may be 
ORTHOGRADE PERFUSION

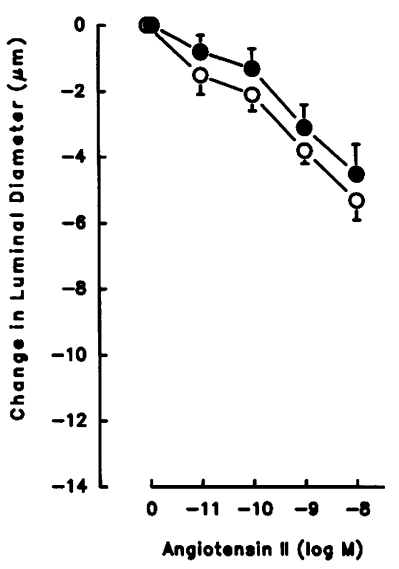

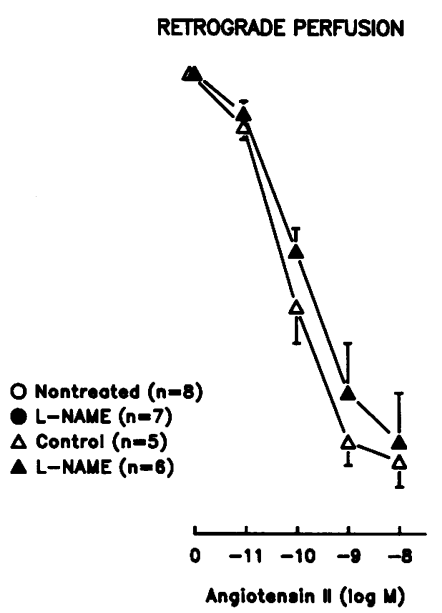

Figure 7. Change in luminal diameter induced by Ang II in nontreated efferent arterioles (Nontreated) and efferent arterioles pretreated with L-NAME. Note that L-NAME had no effect in either orthograde or retrograde perfusion.

partially due to its significant interaction with EDNO in the Af-Art, compared to little, if any, interaction in the Ef-Art.

The reason why L-NAME had less effect on the Ef-Art than on the Af-Art is not clear. It is not likely to be due to insufficient blockade of EDNO synthesis, since L-NAME completely blocked acetylcholine-induced vasodilation in the Ef-Art. Neither is it likely to be due to arteriolar differences in the rate of diffusion of EDNO into underlying vascular smooth muscle cells nor their response to EDNO, since acetylcholine induces similar vasodilation in both Af-Arts and Ef-Arts (26). However, there are several possibilities that could explain our observation. First, it may be that the basal (unstimulated) rate of EDNO synthesis was somehow higher and/or degradation lower in Af-Arts than in Ef-Arts. Second, there is experimental evidence that vascular smooth muscle cells of the Af-Art but not Ef-Art may possess voltage-dependent calcium channels $(27,28)$, and that ENDO could directly hyperpolarize smooth muscle cells of some vessels independently of a putative endothelium-derived hyperpolarizing factor (29). Thus, inhibition of EDNO with L-NAME may have altered membrane poten-

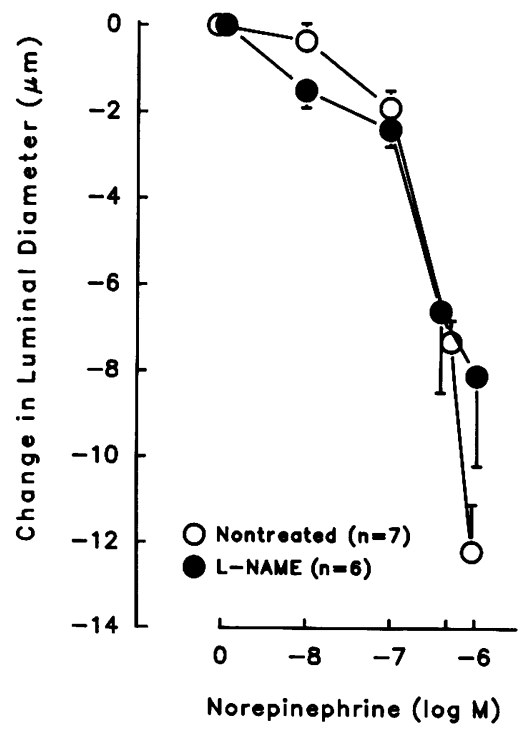

Figure 8. Change in luminal diameter induced by norepinephrine in nontreated afferent arterioles (Nontreated) and afferent arterioles pretreated with LNAME. Note that LNAME had no effect on NE-induced vasoconstriction, in marked contrast to enhanced Ang II action (Figs. 5 and 6).

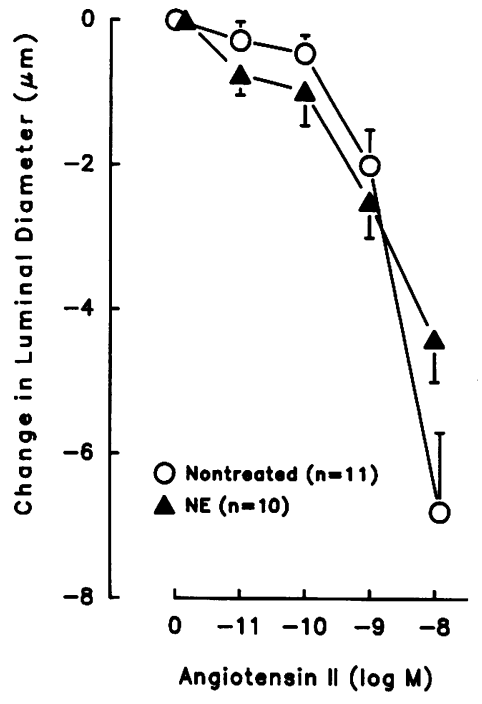

Figure 9. Change in luminal diameter induced by Ang II in nontreated (Nontreated) and norepinephrine-pretreated afferent arterioles $(N E)$. Note that NE had no effect on Ang II-induced vasoconstriction despite a similar decrease in basal diameter. tial, preferentially affecting the Af-Art. Third, EDNO has been shown to modulate myogenic contraction (30). Therefore, LNAME may have potentiated myogenic tone in the Af-Art, but not in the Ef-Art which does not exhibit a myogenic response $(6,7)$. Finally, EDNO have been shown to influence endothelial synthesis of vasoconstrictors such as endothelin (31). Thus, it may be possible that L-NAME increased the level of vasoconstrictors significantly in the Af-Arts but not Ef-Arts.

In our preparation of Af-Arts, the action of both Ang II and endothelin (32) but not NE is augmented by L-NAME. However, the reason for such disparate effects of L-NAME is not clear. It has been proposed that cyclic GMP is involved in the intracellular mechanism of EDNO action (33). It is possible that in the Af-Art, the mechanism of the vasoconstrictor action of both Ang II and endothelin is more susceptible to modulation by intracellular cyclic GMP than that of NE, so that a L-NAME-induced decrease in the basal level of cyclic GMP resulted in enhanced actions of Ang II and endothelin but not NE. It may also be that Ang II and endothelin stimulated EDNO synthesis more than NE under our experimental conditions. In addition, a recent preliminary report suggests that EDNO could displace bound endothelin from its receptors (34), raising the possibility that EDNO may have differential effects on Ang II and endothelin receptors versus NE receptors.

Several studies have recently examined the interaction between Ang II and EDNO in the systemic and renal circulation. De Nicola et al. (35) and Sigmon et al. (36) reported that in anesthetized rats intravenous L-NAME increased systemic blood pressure as well as systemic and renal vascular resistance. The increase in renal vascular resistance was greatly attenuated by either an Ang II antagonist or a converting-enzyme inhibitor (CEI), whereas systemic vascular resistance remained elevated, suggesting a unique interaction between Ang II and EDNO within the kidney. The study of De Nicola et al. (35) has further shown that the interaction takes place not only in the glomerular arterioles but also at the glomerular tuft and proximal tubule. In addition, an interaction between Ang II and EDNO has been shown to occur in the absence of systemic hemodynamic changes in conscious dogs (37). These in vivo findings, taken together with our study strongly suggest that intrarenal EDNO plays an important role in the regulation of renal function by modulating the actions of Ang II. 
Clearance studies in the whole animal showed that acute inhibition of EDNO resulted in decreased renal blood flow with little change in the GFR, suggesting preferential constriction of the postglomerular vessel (Ef-Art) $(38,39)$. Consistent with this observation, a micropuncture study by Zatz and de Nucci (40) showed that intravenous administration of LNAME, which raised mean systemic blood pressure by 30 $\mathrm{mmHg}$, decreased single-nephron plasma flow but had no effect on single-nephron GFR. These changes were associated with marked glomerular hypertension $(73 \mathrm{mmHg}$ in $\mathrm{L}-$ NAME-treated rats versus $50 \mathrm{mmHg}$ in vehicle-treated rats), and calculation of regional vascular resistance indicated a preferential effect on the Ef-Art. Although these in vivo studies show the overall effects of EDNO inhibition, various changes in systemic hemodynamics, hormones and sympathetic nerve activity could have influenced the renal hemodynamic response.

In order to avoid systemic neurohormonal and hemodynamic influences, Radermacher et al. (41) employed a preparation of isolated rat kidney perfused with a synthetic solution at constant pressure. In this preparation, L-NAME decreased the GFR and increased the filtration fraction significantly, suggesting constriction of both Af-Arts and Ef-Arts. However, when red blood cells (which contain hemoglobin that scavenges EDNO) were added to the kidney perfusate, L-NAME caused a similar decrease in the GFR but no change in the filtration fraction, suggesting a predominant effect on the Af-Art. These authors speculated that when the kidneys were perfused with the hemoglobin-free solution, EDNO, which was produced only in the Af-Art and/or glomerulus, reached the Ef-Art through the lumen, whereas addition of red blood cells prevented EDNO from reaching the Ef-Art but not from acting on the luminal side in the Af-Art and/or glomerulus. This would explain why L-NAME increased vascular resistance in both Afand Ef-Arts in the synthetic solution, whereas it had no effect on Ef-Arts in the solution containing red blood cells. These observations are consistent with our study that L-NAME induced a small but significant constriction of the Ef-Art with orthograde perfusion, but had no effect when possible influences of the Af-Art and/or glomerulus was eliminated by perfusing the Ef-Art in a retrograde direction.

In contrast to our results showing the predominant effects of L-NAME on the Af-Art, L-NAME has been shown to produce similar decreases in the luminal diameter of both Af-Arts and Ef-Arts in rat juxtamedullary nephrons perfused with blood in vitro (42). The decreases were markedly attenuated by pretreatment with either a converting-enzyme inhibitor or Ang II antagonist, suggesting interaction between endogenous Ang II and EDNO. However, L-NAME did not augment the vasoconstriction induced by exogenous Ang II, while the sensitivity of the Af-Art and Ef-Art to Ang II was the same in this preparation (17-19). The reason for the discrepancies between these studies and ours is not clear, but may be related to the perfusion solution (synthetic solution versus blood) and/or the presence or absence of macula densa-mediated tubuloglomerular feedback. It may also be possible that in the juxtamedullary preparation, EDNO produced in the Af-Art and/or glomerulus somehow reached the Ef-Art at concentrations high enough to exert its action.

These and previous studies provide evidence that endogenous EDNO regulates Af-Art resistance by itself as well as by modulating the actions of both Ang II and endothelin $(8,32$,
$35,37,40-42$ ). Since the Af-Art is a site of both myogenic and tubuloglomerular feedback responses as well as renin synthesis and secretion (43), alteration of EDNO in the Af-Art would have profound effects on glomerular and systemic hemodynamics, either directly or indirectly by altering renin release (9). Indeed, recent experimental evidence suggests that EDNO may be involved (whether primarily or secondarily) in the altered renal hemodynamics seen in various pathological conditions such as hypertension and ischemic acute renal failure (44). In spontaneously hypertensive rats at a phase of established but uncomplicated hypertension, inhibition of EDNO has been shown to cause exaggerated hypertension compared with their normotensive controls, Wistar-Kyoto rats (45). We have previously shown that L-NAME decreases the luminal diameter of microperfused Af-Arts to a greater degree in spontaneously hypertensive rats than in Wistar-Kyoto rats, suggesting that endogenous EDNO may be high (or intact) in SHR AfArts (46). This activity of EDNO may help maintain glomerular filtration in the face of various structural and functional alterations that favor Af-Art contraction $(47,48)$. On the other hand, EDNO appears to be low in ischemic acute renal failure (49), which is characterized by profound diminution of the GFR that is disproportionate to the observed decrease in renal blood flow (50). It is conceivable that low EDNO may cause a preferential increase in Af-Art resistance by itself as well as by augmenting the actions of various vasoconstrictors, such as Ang II and endothelin which are elevated in ischemic acute renal failure.

In conclusion, our observations clearly demonstrate that sensitivity to Ang II is higher in the isolated microperfused rabbit Ef-Art than in the Af-Art, whereas L-NAME, an inhibitor of EDNO synthesis, causes stronger vasoconstriction in the Af-Art than in the Ef-Art. Furthermore, L-NAME pretreatment augments the vasoconstrictor action of Ang II in the AfArt but not the Ef-Art. Thus endogenous EDNO would appear to modulate Ang II action in the Af-Art but not in the Ef-Art, which may contribute to the difference in sensitivity to Ang II.

\section{Acknowledgments}

This study was supported by National Institutes of Health grants HL28982 and HL46518.

\section{References}

1. Ichikawa, I., and R. C. Harris. 1991. Angiotensin actions in the kidney: renewed insight into the old hormone. Kidney Int. 40:583-596.

2. Davalos, M., N. S. Frega, B. Saker, and A. Leaf. 1978. Effect of exogenous and endogenous angiotensin II in the isolated perfused rat kidney. Am. J. Physiol. 235:F605-F610.

3. Ichikawa, I., J. F. Miele, and B. M. Brenner. 1979. Reversal of renal cortical actions of angiotensin II by verapamil and manganese. Kidney Int. 16:137-147.

4. Kastner, P. R., J. E. Hall, and A. C. Guyton. 1984. Control of glomerular filtration rate: role of intrarenally formed angiotensin II. Am. J. Physiol. 246:F897-F906.

5. Ichikawa, I., J. M. Pfeffer, M. A. Pfeffer, T. H. Hostetter, and B. M. Brenner. 1984. Role of angiotensin II in the altered renal function of congestive heart failure. Circ. Res. 55:669-675.

6. Edwards, R. M. 1983. Segmental effects of norepinephrine and angiotensin II on isolated renal microvessels. Am. J. Physiol. 255:F526-F534.

7. Yuan, B. H., J. B. Robinette, and J. D. Conger. 1990. Effect of angiotensin II and norepinephrine on isolated rat afferent and efferent arterioles. Am. J. Physiol. 258:F741-F750.

8. Ito, S., C. S. Johnson, and O. A. Carretero. 1991. Modulation of angiotensin II-induced vasoconstriction by endothelium-derived relaxing factor in the isolated microperfused rabbit afferent arteriole. J. Clin. Invest. 87:1656-1663. 
9. Romero, J. C., V. Lahera, M. G. Salom, and M. L. Biondi. 1992. Role of the endothelium-dependent relaxing factor nitric oxide on renal function. J. Am. Soc. Nephrol. 2:1371-1387.

10. Ito, S., and O. A. Carretero. 1990. An in vitro approach to the study of macula densa-mediated glomerular hemodynamics. Kidney Int. 38:1206-1210.

11. Moor, P. K., O. A. Al-Swayeh, N. W. S. Chong, R. A. Evans, and A. Gibson. 1990. L- $N^{\mathrm{G}}$-nitro arginine (L-NOARG), a novel, L-arginine-reversible inhibitor of endothelium-dependent vasodilation in vitro. Br. J. Pharmacol. 99:408-410.

12. Palmer, R. M. J., D. S. Ashton, and S. Moncada. 1988. Vascular endothelial cells synthesize nitric oxide from L-arginine. Nature (Lond.). 333:664-666.

13. Click, R. L., W. J. Joyner, and J. P. Gilmore. 1979. Reactivity of glomerular afferent and efferent arterioles in renal hypertension. Kidney Int. 15:109-115.

14. Myers, B. D., W. M. Deen, and B. M. Brenner. 1975. Effects of norepinephrine and angiotensin II on the determinants of glomerular ultrafiltration and proximal tubule fluid reabsorption in the rat. Circ. Res. 37:101-110.

15. Frega, N. S., M. Davalos, and A. Leaf. 1980. Effect of endogenous angiotensin on the efferent glomerular arteriole of rat kidney. Kidney Int. 18:323-327.

16. Hall, J. E., A. C. Guyton, J. E. Jackson, T. G. Coleman, T. E. Lohmeier, and N. C. Trippodo. 1977. Control of glomerular filtration rate by renin-angiotensin system. Am. J. Physiol. 233:F366-F372.

17. Navar, L. G., J. P. Gilmore, W. L. Joyner, M. Steinhausen, R. M. Edwards, D. Casellas, P. K. Carmines, L. B. Zimmerhackl, and S. D. Yokota. 1986. Direct assessment of renal microcirculatory dynamics. Fed. Proc. 45:2851-2861.

18. Casellas, D., P. K. Carmines, and L. G. Navar. 1985. Microvascular reactivity of in vitro blood perfused juxtamedullary nephrons from rats. Kidney Int. 28:752-759.

19. Carmines, P. K., T. K. Morrison, and L. G. Navar. 1986. Angiotensin II effects on microvascular diameters of in vitro blood-perfused juxtamedullary nephrons. Am. J. Physiol. 251:F610-F618.

20. Steinhausen, M., R. B. Sterzel, J. T. Fleming, R. Kuhn, and S. Weis. 1987. Acute and chronic effects of angiotensin II on the vessels of the split hydronephrotic kidney. Kidney Int. 31 (Suppl. 20):S64-S73.

21. Lanese, D. M., B. H. Yuan, S. A. Falk, and J. D. Conger. 1991. Effects of atriopeptin III on isolated rat afferent and efferent arterioles. Am. J. Physiol. 261:F1102-F1109.

22. Dunn, J. M., and L. A. Scharschmidt. 1987. Prostaglandins modulate the glomerular action of angiotensin II. Kidney Int. 31 (Suppl. 20):S-95-S-101.

23. Frangos, J. A., S. G. Eskins, L. V. McIntire, and C. L. Ives. 1985. Flow effect on prostacyclin production by culture human endothelial cells. Science (Wash. DC). 227:1477-1479.

24. Nollert, M. U., E. R. Hall, S. G. Eskin, and L. V. McIntire. 1989. The effect of shear stress on the uptake and metabolism of arachidonic acid by human endothelial cells. Biochim. Biophys. Acta. 1005:72-78.

25. Fleming, J. T., C. Zhang, J. Chen, and J. P. Porter. 1992. Selective preglomerular constriction to nerve stimulation in rat hydronephrotic kidneys. Am. J. Physiol. 262:F348-F353.

26. Edwards, R. M. 1985. Response of isolated renal arterioles to acetylcholine, dopamine, and bradykinin. Am. J. Physiol. 248:F183-F189.

27. Carmines, P. K., and L. G. Navar. 1988. Disparate effects of Ca channel blockade on afferent and efferent arteriolar responses to ANG II. Am. J. Physiol. 256:F1015-F1020.

28. Loutzenhiser, R., K. Hayashi, and M. Epstein. 1989. Divergent effects of $\mathrm{KCl}$-induced depolarization on afferent and efferent arterioles. Am. J. Physiol. 257:F561-F564.

29. Tare, M., H. C. Parkington, H. A. Coleman, T. O. Neild, and G. J. Dusting. 1990. Hyperpolarization and relaxation of arterial smooth muscle caused by nitric oxide derived from the endothelium. Nature (Lond.). 346:69-71.

30. Griffith, T. M., and D. H. Edwards. 1990. Myogenic autoregulation of flow may be inversely related to endothelium-derived relaxing factor activity. Am. J. Physiol. 258:H1171-H1180.
31. Boulanger, C. M., and T. F. Lusher. 1991. Differential effect of cyclic GMP on the release of endothelin-1 from cultured endothelial cells and intact porcine aorta. J. Cardiovasc. Pharmacol. 17 (Suppl. 7):S264-S266.

32. Ito, S., L. A. Juncos, N. Nushiro, C. S. Johnson, and O. A. Carretero. 1991. Endothelium-derived relaxing factor modulates endothelin action in afferent arterioles. Hypertension. 17:1052-1056.

33. Moncada, S., R. M. J. Palmer, and E. A. Higgs. 1992. Nitric Oxide: physiology, pathophysiology, and pharmacology. Pharmacol. Rev. 43:109-142.

34. Goligorsky, M., J. Simone, H. Magazine, T. Anderson, A. Malik, and W. Bahou. 1992. A unique property of nitric oxide (NO) to terminate endothelin-1 (ET-1) signal. J. Am. Soc. Nephrol. 3:544. (Abstr.)

35. De Nicola, L., R. C. Blantz, and F. B. Gabbai. 1992. Nitric oxide and angiotensin II: glomerular and tubular interaction in the rats. J. Clin. Invest. 89:1248-1256.

36. Sigmon, D. H., O. A. Carretero, and W. H. Beierwaltes. 1992. Angiotensin dependence of endothelium-mediated renal hemodynamics. Hypertension. 20:643-650.

37. Alberola, A., F. J. Salazar, T. Nakamura, and J. P. Granger. 1992. Renal hemodynamic effects of angiotensin II (AII): interactions with endothelium derived nitric oxide. FASEB (Fed. Am. Soc. Exp. Biol.) J. 6:A1812. (Abstr.).

38. Lahera, V., M. G. Salom, F. Miranda-Guardiola, S. Moncada, and J. C. Romero. 1991. Effects of $N^{\mathrm{G}}$-nitro-L-arginine methyl ester on renal function and blood pressure. Am. J. Physiol. 261:F1033-F1037.

39. Baylis, C., P. Harton, and K. Engels. 1990. Endothelial derived relaxing factor controls renal hemodynamics in the normal rat kidney. J. Am. Soc. Nephrol. 1:875-881.

40. Zatz, R., and G. de Nucci. 1991. Effects of acute nitric oxide inhibition on rat glomerular microcirculation. Am. J. Physiol. 261:F360-F363.

41. Radermacher, J., B. Klanke, H. J., Schurek, H. F. Stolte, and J. C. Frölich. 1992. Importance of NO/EDRF for glomerular and tubular function: studies in the isolated perfused rat kidney. Kidney Int. 41:1549-1559.

42. Ohishi, K., P. K. Carmines, E. W. Inscho, and L. G. Navar. 1992. EDRFangiotensin II interaction in rat juxtamedullary afferent and efferent arterioles. Am. J. Physiol. 263:F900-F906.

43. Schnermann, J., and J. P. Briggs. 1992. Function of the juxtaglomerular apparatus: control of glomerular hemodynamics and renin secretion. In The Kidney; Physiology and Pathophysiology. D. W. Seldin and G. Giebisch, editors. Raven Press, New York. 1249-1290.

44. Lüscher, T. F., H. A. Bock, Z. Yang, and D. Diederich. 1991. Endothelium-derived relaxing and contracting factors: perspectives in nephrology. Kidney Int. 39:575-590.

45. Lacolley, P. J., S. J. Lewis, and M. J. Brody. 1991. L- $N^{\mathrm{G}}$-nitro arginine produces an exaggerated hypertension in anesthetized SHR. Eur. J. Pharmacol. 197:239-240.

46. Ito, S., and O. A. Carretero. 1992. Impaired response to acetylcholine $(\mathrm{ACH})$ despite intact endothelium-derived relaxing factor (EDRF) in isolated microperfused afferent arterioles (Af-Art) of the spontaneously hypertensive rat (SHR): possible role of endothelium-derived contracting factor (EDCF). J. Hypertens. 10 (Suppl. 4):S4. (Abstr.)

47. Trippodo, N. C., and E. D. Frohlich. 1981. Similarities of genetic (spontaneous) hypertension: man and rat. Circ. Res. 48:309-319.

48. Kato, T., Y. Iwama, K. Okumura, H. Hashimoto, T. Ito, and T. Satake. 1990. Prostaglandin $\mathrm{H}_{2}$ may be the endothelium-derived contracting factor released by acetylcholine in the aorta of the rat. Hypertension. 15:475-481.

49. Conger, J. D., J. B. Robinette, and R. W. Schrier. 1988. Smooth muscle calcium and endothelium-derived relaxing factor in the abnormal vascular responses of acute renal failure. J. Clin. Invest. 82:532-537.

50. Levinsky, N. G. 1977. Pathophysiology of acute renal failure. N. Engl. J. Med. 296:1453-1458. 